\title{
Imagery and organization in memory: Instructional effects
}

\author{
IAN BEGG \\ McMaster University, Hamilton, Ontario, Canada L8S 4KI
}

\begin{abstract}
This paper concerns the relation between mental organization and memory performance. In a series of experiments, it is determined that interactive imagery exceeds separate imagery in cued recall and the organization of free recall. However, the two are indistinguishable in either recognition or the level of free recall unless some additional interunit organization is initially encoded. Although interactive imagery allows a list to be retained as fewer traces than does separate imagery, those traces are necessarily larger. The inverse relation between the number and size of traces is found also in the organization of free recall. This tradeoff results in equivalent total recall.
\end{abstract}

There is no shortage of evidence that imagery instructions are correlated with memory performance. However, the contribution of the research to the understanding of the memory system has been disappointing. For example, Runquist (1973) summarized the impact of the research as "look what we can do with imagery instructions" (p. 295). In a similar vein, Tulving and Madigan (1970) questioned the value of using imagery as an explanatory construct and called for more integration of imagery research with memory theory in general. By 1975, Postman detected some progress along these lines, but concluded that the theoretical efforts of Begg $(1972,1973)$ and Paivio $(1971,1975 a)$ were inching forward slowly at best. The present paper offers a memory-theoretic account of the effects of imagery instructions, with the hope of adequately describing those effects in terms that relate the research to general memory processes. In brief, imaging items together, as opposed to separately, reduces the number of memory units required to retain a given list by increasing the average size of the units. However, any organizational format will be most appropriate for some contexts of use, but will be irrelevant or even antagonistic for other purposes. Consequently, the present paper will be as much concerned with what imagery instructions do not accomplish as with what they do.

Before casting imagery instructions in theoretical terms, let us review the effects of the instructions, based on previous research. In the basic situation of interest, subjects are presented with a list of familiar concrete nouns, randomly paired with each other.

The research reported here was supported by Grant $\mathrm{A} 8122$ from the National Research Council of Canada. I thank the members of the F. T. Bacon Society (Grant Harris, John Mitterer, and Doug Upfold) for considerable support. Reprints can be obtained from Ian Begg, Department of Psychology, McMaster University, Hamilton, Ontario, Canada L8S 4K1.
One group is instructed to form a single image to represent each pair of items interacting in some way, while a second group is instructed to form a separate image for each member of each pair. In subsequent tests of memory, some results are quite intuitive and are easily accommodated by any theory of memory. For example, if one member of each pair serves as a cue for recalling the other, relational imagery exceeds separation imagery (Begg, 1973; Begg \& Anderson, 1976; Bower, 1970; Dempster \& Rohwer, 1974), even if recall is conditionalized on cue recognition (Bower, 1070). Similarly, if pair members are imaged together, they are more likely to be recalled contiguously than if initially imaged separately (Begg \& Anderson, 1976), with equivalent results for noun triplets (Morris \& Stevens, 1974).

However, other results are less intuitive, and may even be surprising. For example, recall cues are no better recognized after relational than separation imagery, whether assessed by hit rate (Bower, 1970) or $\mathrm{d}^{\prime}$ values (Dempster \& Rohwer, 1974). That is, reducing the number of units required to retain a list apparently has no effect on subsequent recognition of list items presented as recall cues. Further, despite the large cued recall difference, relational imagery does not differ from separation imagery in free recall of the same response items (Begg, 1973; Begg \& Anderson, 1976). Even with noun triplets, the instructional differences do not produce different levels of free recall (Hasher, Riebman, \& Wren, 1976; Janssen, 1976, Experiment 3). There is one exception to the latter, since Morris and Stevens (1974) obtained a large recall difference for relational imagery; however, as shown in Experiments 3 and 4 of the present series, their result reflects methodological variables, rather than relational imagery. Overall the concensus is clear. Relational imagery surpasses separation imagery in cued recall and the degree of organization in free recall. The theoretical challenge is thus to construct an account 
that simultaneously allows imagery to be effective where it is effective, but not where it is not.

The present account consists of an encoding theory and a retrieval theory, which will be discussed in turn. The encoding theory proposes that the information contained in a list of words is mentally distributed into some number of memory units, each pertaining to some number of list items. Properly speaking, the meaning of verbal signals does not reside in their physical properties, but rather is produced by whatever knowledge and operations are used by the receiver in interpreting the signals. Some knowledge pertains to perceptual characteristics of objects to which signals such as "truck" refer (cf. Paivio, 1975b); image generation is the process by which that information becomes available for use. Once generated, images can be further processed in various ways; for example, given the pair "railroad-mother," it is possible to generate an image for each constituent, and to integrate those images into a coherent percept of the constituents interacting. Thus, imagery is conceived as an interpretive operation by which nominal items are encoded as memory units. Further, by combining images, it is possible to reduce the number of units to be retained; however, the reduction in number is necessarily accomplished by an increase in the size of the units, defining size as the number of list items to which a unit pertains. On the whole, the encoding theory posits that the memory system distributes a list into some number of units, each of a given size, with the set of all units comprising what is retained regarding the list.

In order to make the preceding discussion more concrete, consider the case in which $\mathrm{N}$ pairs of concrete nouns are presented at a reasonably slow rate. By the present account, each pair will be encoded either as a single unit, with probability $\alpha$, or as two separate units. Thus, the list will be distributed into $\mathrm{N} \alpha$ units, each pertinent to two list items, and $2 \mathrm{~N}(1-\alpha)$ units, each pertinent to one item; an equivalent statement is that the list is retained as $N(2-\alpha)$ memory units, with an average size of $2 /(2-\alpha)$ items/unit. Note that as $\alpha$ increases, there is a decrease in the number of units to be retained, and a concomitant increase in their size. If subjects are specifically instructed to generate a separate image for each item, $\alpha$ could reasonably be expected to be relatively low. In contrast, with instructions to remember each pair as a single interactive image, $\alpha$ should be higher. For example, given a 20-pair list, and respective values of .40 and .75 for $\alpha$, a separation group would retain the list as 32 memory units with an average size of 1.25 items, while a relational group would retain the same list as 25 units with an average size of 1.60 items. The consequence of differential organization for performance depends on task requirements, as specified by the retrieval theory.
The retrieval theory characterizes recall as a staged process in which memory units are contacted, redintegrated, and verbally decoded. By the theory, once some component of a unit is accessed, the entire unit is made available, or redintegrated, if the task requires it. In some tasks, such as recognition, no redintegration is required, since contact per se is sufficient to justify an affirmative decision regarding a test item. However, in both cued and free recall, redintegration of units provides information potentially decodable as additional list items. Cued recall can be analyzed as two major stages. First, the presented cue must be recognized; second, the remainder of the unit must be redintegrated and decoded as the desired response. Since relational and separation imagery do not differ in recognition, the difference in cued recall reflects redintegration and decoding, rather than contact. In a similar fashion, free recall can be analyzed as two major stages. Since recall is assumed to be a process of contacting units, then redintegrating and decoding their contents, the recall protocol should consist of a number of sequences, with items from a given unit recalled contiguously. Therefore, the total number of items recalled can be expressed as the product of the number of sequences and their average length. By the theory, the number of sequences in recall reflects the number of units contacted, while the average length of the sequences reflects the average size of the units. In all, the encoding theory posits that a list is mentally distributed into some number of memory units, each of a given size. The retrieval theory posits that recall is a process in which units are contacted, following which each unit is redintegrated, providing information that may be decoded as list items. In cued recall, contact is reflected by measures of cue recognition, while redintegration and decoding are reflected by measures of recall given cue recognition. In free recall, contact is reflected by measures of the number of sequences of related items, while redintegration and decoding are reflected by measures of the length of these sequences. The next order of business is to relate the hypothetical number and size of memory units to the performance measures outlined.

First, consider recognition of list items. Reducing the number of units necessary for retaining a list could have three possible relations with item recognition. First, if the memory system is limited in terms of the number of units that can be retained, that limit is most likely to be exceeded if lists are distributed into many small units. In such an event, there would be an inverse relation between the number of units and the likelihood that any given unit would be contacted by a test item included in that unit. A second possibility is that combining images results in a loss of discriminability of the constituents; that is, perhaps the mother in an interactive image of "railroad-mother" is less identifiably a mother than a mother imaged alone. In such an event, 
there would be a positive relation between the number of units and the likelihood that any unit would be contacted by a constituent test item. The third logical possibility is that the likelihood of constituent recognition is independent of the number of units formed initially. For example, there might be a limit on the number of units the system can retain, but that limit may be much larger than the number of units in most tasks. With pictures, Standing (1973) has presented up to 10,000 stimuli and has found evidence for an enormous storage capacity. At any rate, there are plausible reasons to expect recognition to be inversely related to the number of memory units, positively related to the number of units, or independent of the number of units.

The experimental evidence that cue recognition is equally good for relational and separation imagery is consistent with the hypothesis that recognition likelihood is independent of the number of units in memory. If the likelihood that a test item will contact its parent unit is independent of the number of units, it is also independent of their size. It also follows that the number of units contacted is a positive function of the number of units initially formed. That is, in order to recognize $90 \%$ of test items, it is necessary to contact more memory units following separation imagery than relational imagery. Quite clearly, as many list items are memorially available following the two tasks, and as many are accessible in recognition. However, since no item can successfully cue another item unless the two are encoded as a single unit, relational imagery exceeds separation imagery in cued recall. It is important that the difference in cued recall does not reflect either the availability of list items in storage or their accessibility overall. Rather, the cued recall procedure, in which one item from each pair appears as a cue, equates the number of units contacted in the two conditions; given an equal number of units contacted, the condition with larger units produces superior recall.

However, in free recall, no such limit is imposed. The most intuitive expectation regarding free recall is that relational imagery will exceed separation imagery, since contacted units are larger. The intuitive expectation is based on the implicit assumption that free recall entails contact with an equal number of units in the different conditions. However, the recognition results indicate that more units are available following separation than relational imagery. Accordingly, one should expect separation imagery to result in a larger number of contacted units, but that the units would be smaller than for relational imagery. In short, one should expect a tradeoff between the number and size of units, with lists retained as many small units being recalled as many short sequences, and lists retained as few large units recalled as fewer, longer sequences. Consequently, the overall level of recall will be relatively unaffected by distribution into more or fewer, smaller or larger units. ${ }^{1}$ At this point, the present experiments will be presented and discussed individually, before turning to more general matters.

\section{Introduction to the Experiments}

This section presents a general overview of the four experiments that comprise the present series. In each experiment, subjects studied lists of pairs or triplets of nouns, imaging the members of each set either separately or together as a single image. Following study, retention was assessed in a variety of ways relevant to specific predictions. In the first experiment, study was followed by one of two test sequences. In one sequence, retention was first assessed by a recognition test in which individual pair members appeared as targets, then by a cued recall test in which one member of each pair served as a cue for the recall of the other; in a second sequence, the order of testing was reversed. As developed above, it was predicted that interactive and separate imagery would result in equivalent recognition performance, regardless of whether the recognition test preceded or followed the recall test; that is, as many list items should be available in memory and accessible in recognition in either case. It was further predicted that the instructions would result in large differences in recall, regardless of whether the recall task preceded or followed recognition testing. That is, the cued recall procedure equates the two conditions in the number of traces contacted, with the result that the larger traces of the interaction condition come into play.

In the second experiment, subjects studied three lists of pairs, again either by interactive or separate imagery. In one condition, the study of each list was followed by a cued recall test; in another condition, the test was free recall. The expectation was that interactive imagery should exceed separate imagery in cued recall but not free recall; in free recall, interactive imagery should result in the recall of longer sequences, but there should be fewer of them than for separation imagery. The experiment also included a delayed test of recall. The delayed test was either free or cued recall, varied orthogonally with the manner of initial test. The prediction was that the instructional conditions would not differ in final free recall, regardless of whether the initial test was free or cued; in contrast, the instructional differences should differ considerably in cued recall, again regardless of the prior test history of the items. Quite simply, retention differences should be observed under conditions of performance that equate the number of contacted units, but not under conditions that allow free retrieval; the conditions do not differ in the number of available items, but rather in the consequences of contact.

The third experiment concerned free recall of noun triplets. Again, it was predicted that both instructional conditions would result in the recall of the same number 
of words, with separate imagery exceeding interactive imagery in the number of triads present in recall, but falling short in the number of words recalled from each triad. The fourth experiment extended the third by including, in addition to separate and interactive imagery, a mnemonic for linking successive triplets to each other. The prediction was that the effect of the mnemonic would be to transform the free recall task functionally to a cued recall task; the subjectively generated cues should again have the effect of equating the conditions in the number of triplets contacted, and hence should produce recall favoring the interactive condition.

\section{EXPERIMENT 1}

The first experiment was a replication and extension of previous research concerning cued recall and recognition of words imaged singly as opposed to interactively. There were two procedural changes over previous research. First, recognition was assessed on a separate test from cued recall, rather than by requiring cue identification on the recall test. Second, recognition was assessed for both cue and response items. In all, subjects studied 60 pairs of common nouns by generating a single image either for each word or for each pair. Half the subjects were tested by item recognition, then were retested for cued recall; the sequence was reversed for the remaining subjects.

By the present account, interactive and separate imagery differ in the composition of the memory system they produce. That is, interactive imagery results in the formation of fewer but larger memory traces than does separate imagery. In recognition, the test item is encoded; that encoding will either contact or fail to contact the trace into which the same nominal item was previously encoded. Such contact depends both on the distinctive similarity (Asch, 1969) of the encoding and trace, and on the availability of the trace. Since neither parameter varies with the number or size of traces, recognition performance should be equivalent for the instructional conditions, regardless of whether recognition precedes or follows cued recall. However, in cued recall, there are two stages. First, the cue must be recognized, a process that should be equally effective for the conditions. Second, the parent trace contacted by the encoded cue must contain information decodable as the desired response; such information is absent in traces that represent single nominal items. Therefore, interactive imagery should markedly exceed separation imagery in cued recall.

\section{Method}

Subjects. Twenty student volunteers were each paid $\$ 2 / \mathrm{h}$; each was tested individually.

Materials and Procedure. A total of 180 nouns were selected from the Paivio, Yuille, and Madigan (1968) norms, with all nouns over 5 in rated imagery and varying widely in other characteristics. The nouns were randomly sorted into 60 pairs and 60 distractors. The 60 pairs were printed on index cards with one noun on the front and one on the back of each card. During study, subjects received each pair for $10 \mathrm{sec}$, with $5 \mathrm{sec}$ for each word. Half the subjects were instructed to form a single image for each presented word during the $5 \mathrm{sec}$ the word was exposed. The other subjects, the interaction group, were instructed to form an image for the first word on each card during the $5 \mathrm{sec}$ the word was exposed, then to incorporate an image of the second word into the image of the first during the $5 \mathrm{sec}$ the second word was exposed. All subjects were instructed to keep every image separate from every other image.

Following study, each subject was tested by recognition followed by cued recall, or the reverse, with each test $6 \mathrm{~min}$ in duration. In the cued test, one member from each pair appeared as a cue for the other; half the cues were the first noun of each pair, half the second. In recognition, there were 60 distractors and 60 old items; 30 of the old items were cues in the alternate test, and 30 were responses, with an equal number of first and second nouns in each case. The test sheet for cued recall thus consisted of 60 words, with a blank space to the left or right of the cue, depending on initial presentation. For recognition, subjects received 120 cards, each with one word printed on it, and sorted the cards into two piles, one old and one new.

\section{Results and Discussion}

Some general conventions adopted throughout the paper are outlined at this point. First, data are presented as proportions to facilitate comparison across experiments, although analyses were conducted on the number correct. Second, means are accompanied by standard deviations in parentheses. Third, reported inferential statistics exceed $\alpha=.05$ unless otherwise indicated. In the first experiment, false alarm rates are not considered, since false alarms were rare (less than $6 \%$ ) and equivalent across conditions.

In the condition in which recognition preceded cued recall, results were precisely as expected. Thus, interactive and separate imagery resulted in equivalent hit rates in recognition, with respective means of .91 $(.05)$ and $.90(.03)$; however, with recall cues provided, interaction markedly exceeded separation in recall, with means of .62 (.14) and .10 (.15) $[\mathrm{t}(9)=5.72]$ Conditionalizing recall on prior recognition resulted in means of .66 and .11, while conditionalizing recognition on later recall produced means of .97 and .97; that is, the instructional conditions differed minimally in overall recognition, or recognition of recalled items, but differed considerably in recall, and recall of recognized items. With cued recall preceding recognition, the results were comparable. Thus, in the initial recall test, interaction exceeded separation, with means of $.51(.22)$ and $.08(.08)[\mathrm{t}(9)=4.17]$, while the respective means of $.93(.05)$ and $.94(.06)$ for recognition did not differ. Recognized items were recalled with means of .54 and .08 , and recalled items were recognized with means of .98 and 1.00 .

Thus the results were as expected. Interaction and separation resulted in indistinguishable recognition performance, regardless of whether the recognition test preceded or followed recall. In contrast, the 
conditions differed enormously in cued recall, again regardless of the placement of the test or the manner of measuring recall. Therefore, the conditions did not differ in the availability of item information, nor in access with information specific to test items. Cued recall differences consequently reflect the organization of information, rather than contact with memory traces.

\section{EXPERIMENT 2}

The second experiment again contrasted interactively and separately imaged pairs in retention tests. Subjects studied and recalled three lists either by cued or free recall. At a later time a final recall test was also administered, with the final test identical to the initial test or changed to the alternate test. The intent of the experiment was again to study the lawful relation between mental organization and memory performance. By the present account, interactive imagery produces fewer but larger traces than separate imagery. Whether such organization is of benefit depends on conditions of performance at the time of test.

First consider immediate cued recall. Cues are equally likely to contact their target traces after either instruction, but the contacted traces are more likely to contain response information for the interactive condition. Accordingly, interaction should exceed separation in cued recall. In free recall, however, the situation is different. From the recognition results, it appears that the amount of available item information does not differ for the instructional conditions. Since separation results in more memory traces than interaction, the number of available traces is a positive function of the number of traces initially formed. Since free recall does not impose a limit on the number of traces accessed, more traces should be accessed for separation than interaction. However, since accessed traces are larger following interaction, interaction should exceed separation in the average length of recall sequences of initially paired items. That is, the recall protocol can be scored for the number of words recalled, the number of pairs from which at least one item is recalled, and the ratio of the two. Since the number of pairs that contribute to recall should be higher for separation than interaction, while the proportion of cases in which both members appear contiguously should favor interaction, the overall level of recall should differ minimally. In short, conditions fostering mental organization as few large units should produce recall of few long sequences, with organization as many small units producing many short sequences.

Finally, consider final recall. By the present account, the difference between conditions in cued recall reflects demands of the performance task in interaction with the structure of mental organization. Accordingly, the difference in cued recall should obtain regardless of whether items were initially acquired and tested by cued or free recall. Conversely, even if items are initially tested by cued recall, later free recall should not differ in the number of items recalled.

\section{Method}

Subjects. Eighty student volunteers each received $\$ 2 / \mathrm{h}$, with 10 in each of eight conditions.

Materials and Procedure. Ninety-six nouns were selected from the Paivio et al. (1968) norms. All nouns had imagery values above 6 and ranged from 6 to 10 letters in length. Half the nouns were high in frequency $(f=A, A A)$, and half were low ( $f<20$ ). Three lists of 16 pairs were constructed. Eight pairs in each list were homogeneous in frequency, with four high and four low; eight pairs were heterogeneous in frequency, with four having a high-frequency first member and four a lowfrequency first member. As in Experiment 1, each pair was printed on an index card, one member on the front and one on the back.

Subjects received one study trial, at the rate of $10 \mathrm{sec} /$ pair, and one test trial $2 \mathrm{~min}$ in duration, for each of the three lists. Instructions and presentation were as in Experiment 1, with subjects reminded of the instructions prior to each list. A short aside is in order. Although it is well known to investigators, it has not been reported in the literature that subjects generally abandon the separation strategy in cued recall; that is, they see how poor their recall is and abandon the strategy. Consequently, the first list is the most likely to reflect accurately the relation between the instruction and performance; analysis will take such factors into consideration. At any rate, half the subjects in each instructional condition were asked to write down as many words or pairs as they cquld remember. The other half were presented with a cue sheet containing the first member of each pair, with instructions to produce the second.

After the third list had been presented and recalled, subjects spent about $1 \mathrm{~h}$ producing oral associates to new sets of nouns or adjectives. Then, half the subjects from each group were asked for free recall of the words from the lists, and half were asked for cued recall. During presentation, half the cues were the first word in the pair and half were the second, with equal numbers of each pair type represented in each cuing condition. The final recall tasks lasted $6 \mathrm{~min}$. Overall, subjects were tested individually in sessions lasting about $1.5 \mathrm{~h}$.

The design was a complete 2 by 2 by 2 factorial, with instructions, method of immediate test, and method of final test as independent factors. Within each combination of factors, the first or second members were of high or low frequency, and within the final cued test, cues were either first or second members. Although all factors were fully analyzed, the Results section ignores word frequency, since the results are replications of other work (e.g., Townsend \& Saltz, 1975), with lowfrequency cues better than high-frequency cues, but with highbetter than low-frequency responses. Similarly, item position (first or second) was ignored, since performance was symmetrical over position.

\section{Results and Discussion}

In the analyses to be reported, means pertain to total performance over all three lists. Results were also analyzed separately by lists, and any discrepancies are pointed out.

Immediate recall tests. Cued recall was considerably better after interaction than separation instructions, with respective mean proportions of $.84(.16)$ and .50 (.30) of the 48 pairs correct $[F(1,38)=21.2]$, as also found by Bower (1970) and by Dempster and Rohwer (1974). However, free recall performance was very similar for the two conditions $(F=1.14)$, with means of $.47(.11)$ and $.43(.13)$ of the 96 words recalled: 
Comparing recall of the second nouns in the cued and noncued conditions, cuing facilitated recall for interaction groups $(F=75.6)$, but not for separation $(F=1.12)$. These results replicate the findings of Begg (1973); interaction imagery exceeded separation imagery in cued recall but not free recall, and cued recall exceeded free recall for interaction imagery but not for separation.

Although the groups did not differ reliably in the number of words recalled in the free recall task, they did differ in the organization of their recall. Interaction groups recalled about twice as many intact pairs as the separation groups, $.40(.17)$ vs. $.21(.15)(F=14.0)$. This corresponds to conditional recall probabilities of .85 and .49 ; note that the probabilities are approximately equal to the cued recall levels of .84 and .50 . In order to contrast the number of chunks recalled, each protocol was rescored, with each word or each intact pair counting as one recalled chunk (cf. Tulving, 1968). Over the three lists, separation resulted in an average of 10.45 (3.17) chunks per list, with interaction resulting in $8.73(1.73)[F(1,38)=4.51]$. Thus, the number of chunks recalled is positively related to the number of traces in memory, and the size of recalled chunks is related to the contents of those traces.

The immediate recall data conform well to the predictions. Thus, interaction instructions increased the level of intrapair organization, relative to separation instructions. Such organization is reflected in the organization of free recall, but not its overall level, and in cued recall. The next sections concern performance in the final recall tasks. The expectations are that the final free recall tasks will produce equivalent levels of recall for the instruction groups, regardless of the performance in initial recall, and that cued recall tasks will yield large effects of the instructions, also regardless of initial recall.

Final free recall. The number of words, of 96 , recalled from the three lists was the dependent measure in a 2 by 2 analysis of variance, with instructions and type of immediate test as independent factors [all $\operatorname{Fs}(1,36)<1]$. For final free recall of initially freely recalled items, interaction and separation instructions led to recall levels of $.31(.10)$ and $.33(.14)$; for items initially tested in cued recall, the means were $.32(.10)$ and $.28(.09)$. Thus, regardless of initial recall differences, which were quite substantial for the cued recall task, final free recall resulted in very similar levels of performance for all groups.

Final cued recall. The dependent measure was the number of words, of 48 , produced in cued recall, with factors as above. For the items initially tested in cued recall, final cued recall levels were $.73(.20)$ and .38 (.25) for interaction and separation groups, with .64 (.20) and .19 (.13) the corresponding means for the groups initially tested in free recall. Interaction clearly exceeded separation $[F(1,36)=39.0]$, and the groups initially cued performed at higher levels on the final test $(F=5.15)$. The latter effect, although present over three lists, was not apparent on performance involving the first list. For that list, initially cued participants recalled $.69(.32)$ and $.19(.25)$ of the items, while $.63(.23)$ and $.18(.21)$ were recalled by those who had initially been tested in free recall. The instructional effect was reliable $[F(1,36)=34.1]$, but no other $F$ values exceeded 1 . Overall, interaction exceeded separation on the final cued test, regardless of initial levels of performance.

Summary. The results are again clear. Interaction exceeds separation both in cued recall and chunk size in free recall, both measures that reflect the contents of recovered traces. However, interaction falls short of separation in the number of chunks recalled. As with the recognition tests, the number of accessible traces is a positive function of the number of traces initially formed. Because of the inverse relation between the number and size of recalled chunks, total recall is equivalent over the different distributions. As with the recognition tests, the results are consistent with the notion of a limited capacity memory system, if the limit is set by the total amount of item information rather than the number of independent traces. It was also determined that the patterns of recall differences depend on the current performance task. Thus, interaction exceeds separation in all cases in which cues are presented, because the cues contact more informative traces in the interaction than the separation case; that is, the number of traces contacted is held constant in cued recall, although generally at a higher level than free recall. In free recall, it is still the case that a revived trace is more informative after interaction than separation processing, as judged by chunk size in recall; however, the number of accessible traces depends on the number of traces formed, so that total recall is relatively unaffected by distribution of list items into memory traces.

\section{EXPERIMENT 3}

In the third experiment, interactive and separate imagery were again contrasted. However, noun triplets constituted the to-be-remembered material. The experiment was conducted for two procedural reasons. First, the organization of recall is more readily measured with triplets than with pairs; as before, it was predicted that separate imagery would result in the recall of more but shorter sequences of related items than would interaction imagery. Second, as discussed in the Introduction, Morris and Stevens (1974) found that interactive imagery for noun triplets not only exceeded separation in the organization of recall, but also in the number of words recalled, while Hasher et al. (1976) and Janssen (1976) found no difference in overall level of recall. In Morris and Stevens' experiments, subjects 
were carefully instructed to keep each image separate from each other image in the separation condition; however, in interaction, they were instructed to form a single image for each triplet of nouns, but were not instructed to keep each compound image separate from each other one. The present experiment instructed subjects appropriately.

\section{Method $\$ 2 / \mathrm{h}$. \\ Subjects. Twenty volunteers in two groups of 10 were paid \\ Materials and Procedure. One hundred and eight concrete nouns with imagery values over 5.6 were selected from the Paivio et al. (1968) norms. The nouns were randomly sorted into three sets of 12 triplets. The words were presented aurally at the rate of $5 \mathrm{sec} /$ word, with an additional $5 \mathrm{sec}$ between triplets. Subjects were instructed to form a single image for each word or each triplet, being careful to keep every image separate from every other one. After each 36-word list, 3 min were allowed for free written recall. After the recall of the third list, 5 min were allowed for recall of all items; data from the final recall test are not reported, since both immediate and final recall produced identical patterns of reliable effects.}

\section{Results and Discussion}

The dependent measure for each analysis reflects total performance over the 108 words in the three lists, since separate analyses by lists produced equivalent results. Recall levels were again equivalent for the instructional conditions, with interaction yielding recall of .51 (.13) of the items, and separation, .50 (.11). Therefore, with careful instructions, the groups become equivalent in recall performance. Despite equivalence in overall recall, the groups differed in the organization of recall. Of the 36 triplets, at least one item was recalled from $.62(.13)$ for interaction, and $.75(.08)$ for separation $[F(1,18)=6.39]$; this measure is more conservative than the measure used in Experiment 2, but the difference still obtains. Chunk size in recall was measured leniently by the number of words from a triplet that appeared anywhere in recall, given at least one, and stringently by the repetition ratio (RR) (Frender \& Doubilet, 1974), which requires contiguous recall. Interaction exceeded separation on both measures, with respective mean words per triplet of $2.48(.34)$ and $2.01(.30)[F(1,18)=10.6]$, and $R R$, with means of $.52(.13)$ and $.32(.14)(\mathrm{F}=11.8)$.

Clearly, the results are exactly as expected. Since interaction and separation differ in opposite directions in the number and length of recalled sequences, they do not differ in the number of words recalled. Once again, parameters of mental organization are mirrored in measures of recall organization.

\section{EXPERIMENT 4}

The fourth experiment replicated and pursued further the results of Experiment 3. Since Morris and Stevens' (1974) results were assumed to reflect intertriplet organization, a mnemonic condition was introduced to provide such organization. Many mnemonic techniques are available for linking successive units of information. The mnemonic chosen was a variant of the method of loci (cf. Paivio, 1971), in which each image is mentally located at a particular locus that is mentally recoverable during retrieval. Specifically, subjects pictured each triplet at a numerical location on a clock face, allowing a simple retrieval strategy. The effect of the mnemonic should be to equate the conditions in the number of triplets contacted during retrieval, hence should favor interaction in the number of words recalled. Another effect of the mnemonic should be to reduce the difference between the conditions in organization of recall; thus, if each separately imaged word in a triplet is located at a single locus, that locus is a cue for each word, with the result that the words may be recalled contiguously without having been remembered as a single image.

\section{Method}

Subjects. Forty volunteers were paid $\$ 2 / \mathrm{h}$.

Materials and Procedure. The same lists as in Experiment 3 served as material for four groups of subjects. Two groups of subjects, the control conditions, received either interaction or separation instructions as in Experiment 3. Two remaining groups received the same instructions, but in addition, they were shown a large clock face with three sides of a square drawn outside the face next to each numeral. Subjects were instructed either to remember each triplet as a single image within the square next to each numeral, or to locate an image of each word in a different side of the square. Presentation rate and testing were as in Experiment 3 . Subjects were tested individually.

\section{Results and Discussion}

In overall recall, interaction and separation were equivalent in the control condition, with means of .57 (.11) and $.53(.15)$. In the mnemonic condition, interaction exceeded separation, with means of .63 (.17) and .49 (.15) $[\mathrm{F}(1,18)=3.85, \mathrm{p}=.07]$. In the number of triads represented in recall, separation exceeded interaction in the control condition, with means of $.77(.12)$ and $.63(.10)$, but the two did not differ with the mnemonic, .71 (.15) and .73 (.17), resulting in a reliable interaction $[F(1,36)=4.20]$. In the number of words per recalled triplet, interaction exceeded separation $[\mathrm{F}(1,36)=40.0]$, with means of $2.74(.22)$ and $2.03(.32)$ for the control condition, and 2.52 (.32) and 2.04 (.33) for the mnemonic. Similarly, RR showed strong effects of instructions $[F(1,36)=50.0]$ and an interaction between the variables $[F(1,36)=5.51]$. Interaction exceeded separation in the mnemonic condition, with means of $.56(.08)$ and $.40(.11)$, and by a greater amount in the control condition, with means of $.62(.07)$ and .30 (.15).

The results again accord well with expectations. Although interaction and separation differ minimally in overall recall, providing an interunit organization benefits the interaction group by raising the number of 
units contacted. With separation, the effect of the interunit mnemonic is to increase the organization of recall, although not to the level of the interaction condition.

\section{GENERAL DISCUSSION}

The results of the present experiments are consistent with previous research and extend imagery research in a number of ways. The primary assumption underlying the research is that lists studied by interactive imagery are remembered as fewer but larger traces than lists studied by separate imagery; the conditions do not differ in the amount of information preserved, but rather in its organization. Since the conditions do not differ in the amount of available information, they do not differ in the probability that a list item will be recognized, as also found by Bower (1970) and Dempster and Rohwer (1974). Similarly, if organization is manipulated by presenting categorized nouns, recognition performance does not vary (see Kintsch, 1970 , p. 248). Further, it was determined in the present series that the equivalence in recognition holds for both cue items and response items tested before recall, or tested after the recall test, and that effective cues and recalled responses are also equally well recognized for the instructional conditions. Accordingly, one can draw the strong conclusion that interactive and separate imagery do not yield memory traces for different numbers of list items. An exactly equivalent statement is that the number of accessible traces is a positive function of the number of traces initially formed, and that the number of items accessible given contact with a trace is a positive function of the number of items encoded in that trace initially.

The results of cued recall tests in the present series showed marked advantages for interactive imagery over separate imagery, in agreement with previous research (Begg, 1973; Begg \& Anderson, 1976; Bower, 1970; Dempster \& Rohwer, 1974). Since the conditions do not differ in the number of recognizable cues, the entire difference in performance reflects the contents of contacted traces; if a recognized cue is the only constituent of a trace, recall cannot succeed. That a cue cannot provide access to information unless it is initially encoded with that information was first pointed out by Locke (1690, cited by Mandler \& Mandler, 1964, p. 34), and has more recently become the familiar principle of encoding specificity (Tulving \& Thomson, 1973), or the phenomenon of limited access (Spyropoulos \& Ceraso, 1977). In short, the present contention is not without precedent. There are also many cases in studies of organization in which cuing facilitates recall by increasing the number of units contacted, but leaves the number of items recalled per unit invariant ( $\mathrm{cf}$. Tulving \& Pearlstone, 1966: Tulving \& Psotka, 1971). The present result is thus not peculiar to imagery instructions.
In free recall, the present findings are somewhat more complex than is the case for recognition or cued recall, both of which limit the number of traces contacted to the number of test items presented. The first result is that the number of units contributing to recall is a positive function of the number of units formed; separate imagery results in more memory traces than interaction imagery, and also results in more chunks in recall. Even with single word units, it has been found that the number of words recalled is a positive function of the number presented in a given time; for example, with a total time of $80 \mathrm{sec}$, subjects recall 9.07 of 10 words, 13.90 of 20 , and 15.53 of 40 (Roberts, 1972, auditory condition). Again, the result pertains to more than imagery.

The second result is an extremely general one; items remembered as a single unit tend to be recalled contiguously, with the result that interactive imagery exceeds separate imagery in the organization of recall. As Locke described it, "the whole gang, always inseparable, show themselves together" (Mandler \& Mandler, 1964, p. 41). More recently, Mandler (1968) referred to "the integrated or organized response sequence... that runs off automatically once it was initiated" (p. 109), while Tulving (1968) described an "integrated and invariant response... readily completed if a substantial part of it is given" (p.7). Experimentally, many manipulations designed to increase the size of memory units have been shown to increase organization in recall; examples include interitem association (Jenkins, Mink, \& Russell, 1958), categorization (Bousfield, 1953), frequency (Deese, 1959, 1960), and approximations to English (McNulty, 1966).

The third result is that total recall is relatively unaffected by the number or size of independent memory traces; since increments in size necessarily entail decrements in number, a tradeoff ensues. Few studies have manipulated only mental distribution of a given list. Gianutsos (1972) and Murdock and Carey (1972) presented constant-length lists grouped in various fashions and found grouping to have little effect on recall, with that effect restricted to recently presented items. In experiments in my lab, Robertson (1975) presented lists of 12 items singly, as six groups of 2 items, four groups of 3 , three groups of 4 , or two groups of 6 . The general finding was that recall was invariant over grouping, because the number of groups present in recall increased with the number of initial groups, and the number of items per recalled group increased with the size of the groups. Earhard (1967) also found no difference in free recall as a function of the number or size of sets into which a list was distributed, although cued recall increased with the number of sets. In the imagery studies reported here and elsewhere (Begg, 1973; Begg \& Anderson, 1976), interactive and separate imagery do not differ in total recall. 
Using a line of reasoning like that developed here, Begg and Young (1977) studied the form-class effect. Children show better cued recall for noun pairs linked by verbs than the same pairs linked by conjunctions (the giant hears the boat vs. the giant and the boat). If that effect is entirely organizational, then (1) the effect should not obtain in free recall; (2) the effect in cued recall should disappear if interactive imagery instructions are given, since conjunctions should be raised to the level of verbs; (3) the effect should also disappear with separation instructions, but because verbs are lowered to the level of conjunctions; and (4) the effect should obtain within a form class, such as prepositions, if prepositions implying contact (in, on) are contrasted with prepositions more suggestive of separation (near, by). All the predicted results obtained.

Finally, it was found that providing a means of linking traces to each other increases the number of traces contacted in recall. Intertrace organization is the mechanism posited by Mandler (1968) to account for increasing recall as a function of the number of categories into which a list is subjectively sorted (Basden \& Higgins, 1972; Mandler, 1967; Melkman, 1975), and studied experimentally by Bower, Clark, Lesgold, and Winzenz (1969). The result was extended in my lab (Sestokas, 1974). Fifteen sets of five concrete nouns were studied and recalled, followed by a final recall test. In a control condition, each set was studied for memory. In one organization condition, subjects produced a short narrative linking the five nouns of each set together, but keeping each set separate. In a continuous organization condition, subjects also produced a narrative for each set, but continued the narrative across sets. Although both organization conditions exceeded control in the number of words recalled per recalled set, only the continuous condition exceeded control in the number of sets recalled. Thus, intertrace and intratrace organization are separately manipulable, the former affecting the number of traces contacted, the latter affecting the number of items recalled from each trace.

The purpose of this paper has been to demonstrate the lawful effects of imaginal organization on memory performance, to account for those results in terms of memory-theoretic terms, and to show that the results reflect general organization rather than some peculiarity of imagery. I conclude that imagery research can contribute to and benefit from general advances in memory theory.

\section{REFERENCES}

Asch, S. E. A reformulation of the problem of associations. American Psychologist, 1969, 24, 92-102.

BASDEN, D. R., \& Higgins, J. Memory and organization: Category recall. and retrieval capacity. Journal of Verbal Learning and Verbal Behavior, 1972, 11, 157-163.
BEGG, I. Recall of meaningful phrases. Journal of Verbal Learning and Verbal Behavior, 1972, 11, 431-439.

BEGG, I. Imagery and integration in the recall of words. Canadian Journal of Psychology, 1973, 27, 159-167.

BEGG, I., \& ANDERson, M. C. Imagery and associative memory in children. Journal of Experimental Child Psychology, 1976, 21, 480-489.

BegG, I., \& Young, B. J. An organizational analysis of the form-class effect. Journal of Experimental Child Psychology, 1977, 22, 503-519.

Bousfield, W. A. The occurrence of clustering in the recall of randomly arranged associates. Journal of General Psychology, 1953, 49, 229-240.

Bower, G. H. Imagery as a relational organizer in associative learning. Journal of Verbal Learning and Verbal Behavior, $1970,9.529-533$.

Bower, G. H., Clark, M. C., Lesgold, A. M., \& Winzenz, D. Hierarchical retrieval schemes in recall of categorized word lists. Journal of Verbal Learning and Verbal Behavior, 1969, 8, 323-343.

DEESE, J. Influence of inter-item associative strength upon immediate free recall. Psychological Reports, 1959, 5, 305-312.

DEESE, J. Frequency of usage and number of words in free recall: The role of associations. Psychological Reports, 1960 , 7, 337-344.

Demster, R. N., \& Rohwer, W. D. Component analysis of the elaborative encoding effect in children's learning. Journal of Experimental Psychology, 1974, 103, 400-408.

EARHARD, M. Cued recall and free recall as a function of the number of items per cue. Journal of Verbal Learning and Verbal Behavior, 1967, 6, 257-263.

Frender, R., \& DoubiLet, P. More on measures of category clustering in free recall-although probably not the last word. Psychological Bulletin, 1974, 81, 64-66.

Gianutsos, R. Free recall of grouped words. Joumal of Experimental Psychology, 1972, 95, 419-428.

HASher, L., RiebMan, B., \& WREN, F. Imagery and the retention of free-recall learning. Journal of Experimental Psychology: Human Learning and Memory, 1976, 2, 172-181.

JANSSEN, W. In the nature of the mental image. Soesterberg, The Netherlands: Institute for Perception TNO, 1976.

Jenkins, J. J., Mink, W. D., \& Russell, W. A. Associative clustering as a function of verbal associative strength. Psychological Reports, 1958, 4, 127-136.

KINTsCH, W. Learning, memory and conceptual process. New York: Wiley, 1970.

Mandler, G. Organization and memory. In K. W. Spence \& J. T. Spence (Eds.), The psychology of learning and motivation: Advances in research and theory (Vol. 1). New York: Academic Press, 1967.

Mandler, G. Association and organization: Facts, fancies, and theories. In T. R. Dixon \& D. L. Horton (Eds.), Verbal behavior and general behavior theory. Englewood Cliffs, N.J: Prentice-Hall, 1968.

MANDler, J. M., \& MANdler, G. Thinking: From association to gestalt. New York: Wiley, 1964.

Martin, E., Fleming, F. G., Hennrikus, D. J., \& Erickson, E. A. Studies of the length-difficulty relation in serial memorization. Joumal of Verbal Learning and Verbal Behavior, 1977, 16, 535-548.

McNultY, J. A. The measurement of "adopted chunks" in free-recall learning. Psychonomic Science, 1966, 4, 71-72.

Melkman, R. Effects of preferred and imposed number of categories on recall. Joumal of Experimental Psychology: Human Learning and Memory, 1975, 1. 280-285.

Morris, P. E., \& Stevens, R. Linking images and free recall. Journal of Verbal Learning and Verbal Behavior, 1974, 13, 310-315.

Murdock, B. B., JR., \& CAREY, S. T. Release from interference in single-trial free recall. Journal of Verbal Learming and Verbal Behavior, 1972, 11, 398-402. 
PaIvio, A. Imagery and verbal processes. New York: Holt. Rinehart, \& Winston, 1971.

PaIvio, A. Coding distinctions and repetition effects in memory. In G. H. Bower (Ed.). The psychology of learning and motivation (Vol. 9). New York: Academic Press, 1975. (a)

Paivio, A. Perceptual comparisons through the mind's eye. Memory \& Cognition, 1975, 3. 635-647. (b)

Paivio, A., Yuille, J. C.. \& Madigan, S. A. Concreteness, imagery and meaningfulness values for 925 nouns. Journal of Experimental Psychology Monograph Supplement, 1968 , 76(1, Pt. 2).

Postman, L. Verbal learning and memory. Annual Review of Psychology, 1975, 26, 235-334.

ROBERTs, W. A. Free recall of word lists varying in length and role of presentation: A test of the total-time hypothesis. Journal of Experimental Psychology, 1972, 92, 365-372.

ROBERTSON, R. A. Concreteness and grouping in organized memory. Unpublished master's thesis, McMaster University, 1975.

RunQuist, W. N. Aspects of cognitive process: A review of imagery and verbal learning by Allan Paivio. Canadian Psychologist, 1973, 14, 290-296.

SESTOKAs, A. Narrative instructions and concreteness in free recall. Unpublished honors thesis, McMaster University, 1974.

SpYropoulos, T., \& Ceraso, J. Categorized and uncategorized attributes as recall cues: The phenomenon of limited access. Cognitive Psychology, 1977, 9, 384-402.

Standing, L. G. Learning 10.000 pictures. Quarterly Journal of Experimental Psychology, 1973, 25, 207-222.

Townsend, D. J., \& Sal.Tz, E. The effects of stimulus frequency on subsequent recall of backward associates. Cunadian Journal of Psychology, 1975, 29, 3-10.
Tulving. E. Theoretical issues in free recall. In T. R. Dixon \& D. L. Horton (Eds.), Verbal behavior and general behavior theory. Englewood Cliffs, N.J: Prentice-Hall, 1968.

Tulving, E., \& Madigan, S. A. Memory and verbal learning. Annual Review of Psychology, 1970, 21, 437-484.

Tulving, E., \& Pearlstone, $Z$. Availability versus accessibility of information in memory for words. Joumal of Verbal Learning and Verbal Behavior, 1966, 5, 381-391.

Tulving, E., \& PsotkA, J. Retroactive inhibition in free recall: Inaccessibility of information available in the memory store. Journal of Experimental Psychology, 1971, 87, 1-8.

Tulving, E., \& Thomson, D. M. Encoding specificity and retrieval processes in episodic memory. Psychological Review, 1973, 80, 352-373.

\section{NOTE}

1. A similar point has been made by Martin with regard to serial recall: "For a given type of material, list length is the sole determiner of difficulty. The tradeoff between number of subsequence members and number of subsequences is exact; what one gains by subdividing a list into smaller groups is precisely what one loses in having to handle more groups" (Martin, Fleming, Hennrikus, \& Erickson, 197\%, p. 545).

(Received for publication October 21, 1977; revision accepted January 6,1978 .) 